Original Article

\title{
DESIGN, SYNTHESIS AND ANTIMICROBIAL STUDIES OF 5-BENZYLIDENE SUBSTITUTED RHODANINE CONTAINING HETEROCYCLES
}

\author{
CICI MATHEW ${ }^{a^{*}}$, BINDU SARASWATI ${ }^{b}$, NAND LAL $^{b}$, JOYAMMA VARKEYa $^{a}$
}

aCollege of Pharmaceutical Sciences, Government Medical College, Thiruvananthapuram 695011 (India), bCorporate Research and Development Centre, HLL Lifecare Limited, Thiruvananthapuram 695017 (India)

*Email: ciciabi@gmail.com

Received: 29 Oct 2020, Revised and Accepted: 09 Mar 2021

ABSTRACT

Objective: The principal objective of the study was to synthesize and evaluate the biological activities of a novel class of 5-benzylidene substituted rhodanine derivatives as antimicrobial agents.

Methods: All the synthesized compounds (D1-D10) were screened for their antimicrobial activities using microdilution methods as per the reported procedure. All compounds were evaluated as potential antimicrobial agents against gram-positive bacteria: Bacillus cereus, Staphylococcus aureus, gram negative bacteria: Escherichia coli Pseudomonas aeruginosa and Klebsiella pneumoniae Fungal cultures used in the study were Aspergillus niger, Candida albicans, Candida parapsilosis, Candida tropicalis and Candida glabrata.

Results: Compound D6 showed good antifungal activity in the MIC range $16 \mu \mathrm{g} / \mathrm{ml}$ against Candida tropicalis and Compound D10 showed good antifungal activity in the MIC range $16 \mu \mathrm{g} / \mathrm{ml}$ against Candida glabrata. Compounds D2 and D5 showed good antibacterial activity at $32 \mu \mathrm{g} / \mathrm{ml}$. all the other compounds showed moderate antibacterial activity.

Conclusion: Based on the above results, it can be concluded that the compounds may lead to the development of more potent antimicrobial drug candidates in the near future.

Keywords: Anti-fungal, Rhodanine, Microdilution, Anti-bacterial

(C) 2021 The Authors. Published by Innovare Academic Sciences Pvt Ltd. This is an open access article under the CC BY license (https://creativecommons.org/licenses/by/4.0/) DOI: https://dx.doi.org/10.22159/ijpps.2021v13i5.40106. Journal homepage: https://innovareacademics.in/journals/index.php/ijpps.

\section{INTRODUCTION}

Design, synthesis and development of pharmaceutically active molecules have always been a primary objective of medicinal chemistry. Treatment of infectious diseases is a challenging task for researchers and due to the increasing number of multidrug-resistant microbial pathogens, the discovery of new molecules to combat drug resistance is always a necessity [1-6]. The existing antimicrobial drugs for the treatment of infectious diseases are insufficient to protect us for the long term because of an increasing number of resistant strains. Hence there is an emergent need for the development of newer antimicrobial agents with a new mode of action. Rhodanines are accepted as advantaged heterocycles in medicinal chemistry as one of the 4-thiazolidinones subtypes [7-11]. Thioxo-thiazolidinone (Rhodanine) and its derivatives $[12,13]$ are reported to have a broad spectrum of biological activities such as antibacterial [14-19], antifungal [20-22], anti-diabetic [23, 24], anti-tubercular [25-27] anticancer [28, 29] anti-HIV [30-38] and antimalarial [39]. Rhodanine derivatives have been investigated for Alzheimer's disease also [40, 41]. Rhodanine (2-thioxothiazolidin-4-one) can be used for chemical modifications such as N-3 and/or "active methylene" C-5 substitution can be found that are capable of generating potential for new bioactive compounds $[42,43]$. We, therefore, took up designing new chemical entity of 3-[(dialkylamino) alkyl]-2-thioxothiazolidinone (fig. 1) by substituting various benzylidene derivatives at $5^{\text {th }}$ position and the evaluation of their in vitro antimicrobial activity. In this study, we report in vitro activity of some very potent 3-(dialkylamino) alkyl substituted rhodanine derivatives.

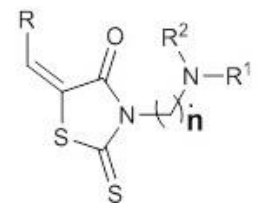

Fig. 1: General structure of designed molecular framework

\section{MATERIALS AND METHODS}

\section{Synthesis}

All the chemicals (reagent grade) were obtained from commercial sources and were used as supplied without further purification. Melting points were determined in open capillary tubes on an electrically heated block and are uncorrected. The reaction progress and purity of the synthesized compounds were monitored by analytical thin-layer chromatography (TLC) on pre-coated silica gel plates (Merck India Ltd). IR spectra $\left(v_{\max }\right.$ in $\left.\mathrm{cm}^{-1}\right)$ of the compounds were recorded on Perkin Elmer's FT-IR RX1 PC spectrophotometer. ${ }^{1} \mathrm{H}$ NMR and ${ }^{13} \mathrm{C}$ NMR spectra were recorded on Bruker AVANCE III spectrometers (operating at $500 \mathrm{MHz}$ for ${ }^{1} \mathrm{H}$, and $125 \mathrm{MHz}$ respectively for ${ }^{13} \mathrm{C}$ ) in deuterated solvents with TMS as internal reference (chemical shifts $\delta$ in ppm). Electron Spray Ionization Mass spectra (ESI-HRMS) were recorded on Thermo Scientific Exactive plus Orbitrap spectrometer. All spectral analysis data were under the assigned structures. All the compounds were characterised by TLC, IR, ${ }^{1} \mathrm{H}$ and ${ }^{13} \mathrm{C}$ NMR, and HRMS. All the chemicals and solvents were procured from Sigma-Aldrich/Merck India Ltd. 5-benzylidene substituted Rhodanine containing heterocycles were synthesized as per the reported procedure.

Different derivatives were synthesized (table 1) by the reaction of $\mathrm{N}$ substituted Rhodanine (1), substituted benzaldehyde (2) and ammonium acetate in a minimum amount of acetic acid [44]. Stirred and refluxed the mixture at $80-85^{\circ} \mathrm{C}$. TLC was checked. After completion of the reaction, the reaction mixture was washed with ethyl acetate and dried in a rota vapour to get (3) in good to excellent yield

5-Benzylidene-3-(2-(dimethylamino) ethyl)-2-thioxothiazolidin -4-one (D1)

The title compound was synthesized from 3-(2-(dimethylamino) ethyl)-2-thioxothiazolidin-4-one $(0.5 \mathrm{~g}, 0.0024 \mathrm{~mol})$, benzaldehyde $(0.24 \mathrm{ml}, 0.0024 \mathrm{~mol})$ and ammonium acetate $(0.37 \mathrm{~g} .0 .0048 \mathrm{~mol})$ in a minimum amount of acetic acid. Stirred and refluxed the reaction 
mixture at $80-85{ }^{\circ} \mathrm{C}$ for $1.5 \mathrm{~h}$. The reaction was monitored with TLC. After completion of the reaction, the reaction mixture was washed with ethyl acetate and the residue was dried under reduced pressure. Then the residue was dissolved in chloroform and washed with water. The chloroform layer was evaporated and then again, it was dissolved in a minimum amount of chloroform $(5 \mathrm{ml})$. It was kept in a freezer overnight. Brown coloured solid was obtained, which was separated by filtering it with the help of sintered crucible.

Table 1: Structures of newly designed compounds (D1-D10)

\begin{tabular}{|c|c|c|}
\hline Code & Structure & Name \\
\hline D1 & & 5-Benzylidene-3-(2-(dimethyl amino)ethyl)-2-thioxothiazolidin-4-one \\
\hline D2 & & $\begin{array}{l}\text { 3-(2-(dimethyl amino) ethyl)-5-(4-ethylbenzylidene)-2- } \\
\text { thioxothiazolidin-4-one }\end{array}$ \\
\hline D3 & & $\begin{array}{l}\text { 3-(2-(dimethyl amino) ethyl)-5- } \\
\text { (4-methylbenzylidene)-2-thioxothiazolidin-4-one }\end{array}$ \\
\hline D4 & & $\begin{array}{l}\text { 3-(2-(diethylamino)ethyl)-5- } \\
\text { (4-ethylbenzylidene)-2-thioxothiazolidin-4-one }\end{array}$ \\
\hline D5 & & $\begin{array}{l}\text { 3-(2-(dimethylamino)ethyl)-5- } \\
\text { (2-methylbenzylidene)-2-thioxothiazolidin-4-one }\end{array}$ \\
\hline D6 & & $\begin{array}{l}\text { 3-(2-(dimethylamino)ethyl)-5- } \\
\text { (4-isopropylbenzylidene)-2-thioxothiazolidin-4-one }\end{array}$ \\
\hline D7 & & $\begin{array}{l}\text { 5-benzylidene-3-(2-(diethylamino)ethyl) } \\
\text {-2-thioxothiazolidin-4-one }\end{array}$ \\
\hline D8 & & $\begin{array}{l}\text { 3-(2-(diethylamino)ethyl)-5- } \\
\text { (4-methylbenzylidene)-2-thioxothiazolidin-4-one }\end{array}$ \\
\hline D9 & & $\begin{array}{l}\text { 3-(2-(diethylamino)ethyl)-5- } \\
\text { (2-methylbenzylidene)-2-thioxothiazolidin-4-one }\end{array}$ \\
\hline D10 & & $\begin{array}{l}\text { 3-(2-(diethylamino)ethyl)-5- } \\
\text { (4-isopropylbenzylidene)-2-thioxothiazolidin-4-one }\end{array}$ \\
\hline
\end{tabular}


3-(2-(dimethylamino) ethyl)-5-(4-ethylbenzylidene)-2-thioxothiazolidin-4-one (D2)

The title compound was synthesized as per the procedure for compound D1. 3-(2-(dimethylamino) ethyl)-2-thioxothiazolidin-4one $(0.5 \mathrm{~g}, 0.0024 \mathrm{~mol})$, 4-ethylbenzaldehyde $(0.328 \mathrm{ml}, 0.0024 \mathrm{~mol})$, and ammonium acetate $(0.369 \mathrm{~g}, 0.0048 \mathrm{~mol})$ in minimum amount of acetic acid was taken as reaction mixture.

\section{3-(2-(dimethylamino)ethyl)-5-(4-methylbenzylidene)-2- thioxothiazolidin-4-one (D3)}

The title compound was synthesized as per the procedure for compound D1 by taking 3-(2-(dimethylamino)ethyl)-2-thioxothiazolidin-4-one $(0.5 \mathrm{~g}, 0.0024 \mathrm{~mol}), 4$-methylbenzaldehyde $(0.254$ $\mathrm{ml}, 0.00215 \mathrm{~mol})$ and ammonium acetate $(0.37 \mathrm{~g}, 0.0048 \mathrm{~mol})$ in minimum amount of acetic acid.

\section{3-(2-(diethylamino)ethyl)-5-(4-ethyl benzylidene)-2-thioxo- thiazolidin-4-one (D4)}

The title compound was synthesized as per the procedure for compound D1 by taking 3-(2-diethylamino) ethyl-2thioxothiazolidine-4-one $(0.5 \mathrm{~g}, 0.0021 \mathrm{~mol})$, 4-ethylbenzaldehyde $(0.28 \mathrm{ml}, 0.0021 \mathrm{~mol})$ and ammonium acetate $(0.324 \mathrm{~g}, 0.0042 \mathrm{~mol})$ in minimum amount of acetic acid.

\section{3-(2-(dimethylamino)ethyl)-5-(2-methylbenzylidene-2- thioxothiazolidin-4-one (D5)}

The title compound was synthesized as per the procedure for compound D1 by taking 3-(2-(diethylamino)ethyl)-2-thioxothiazolidin-4-one $(0.5 \mathrm{~g}, 0.0024 \mathrm{~mol}), 2$-methylbenzaldehyde $(0.277$ $\mathrm{ml}, 0.0024 \mathrm{~mol})$ and ammonium acetate $(0.37 \mathrm{~g}, 0.0048 \mathrm{~mol})$ in minimum amount of acetic acid.

\section{3-(2-(dimethylamino)ethyl)-5-(4-isopropylbenzylidene)-2- thioxothiazolidin-4-one (D6)}

The title compound was synthesized as per the procedure for compound D1 by taking 3-(2-(dimethylamino)ethyl)-2thioxothiazolidin-4-one $(0.5 \mathrm{~g}, 0.0024 \mathrm{~mol})$, isopropyl benzaldehyde $(0.356 \mathrm{~g}, 0.0024 \mathrm{ml})$, and ammonium acetate $(0.37 \mathrm{~g}, 0.0048 \mathrm{~mol})$ in minimum amount of acetic acid.

\section{5-benzyliden-3-(2-diethylamino)ethyl)-2-thioxothiazolidin-4- one (D7)}

The title compound was synthesized as per the procedure for compound D1 by taking 3-(2-(diethylamino)ethyl)-2thioxothiazolidin-4-one $(0.5 \mathrm{~g}, 0.0021 \mathrm{~mol})$, benzaldehyde $(0.21 \mathrm{ml}$, $0.0021 \mathrm{~mol})$, and ammonium acetate $(0.3237 \mathrm{~g}, 0.0042 \mathrm{~mol})$ in minimum amount of acetic acid.

\section{3-(2-(diethylamino)ethyl)-5-(4-methylbenzylidene)-2-thioxo- thiazolidin-4-one (D8)}

The title compound was synthesized as per the procedure for compound D1 by taking 3-(2-(diethylamino)ethyl)-2-thioxothiazolidin-4-one $(0.5 \mathrm{~g}, \quad 0.0021 \mathrm{~mol}), \quad 4$-methylbenzaldehyde $(0.252 \mathrm{~g}, 0.0021 \mathrm{~mol})$, and ammonium acetate $(0.32 \mathrm{~g}, 0.0042 \mathrm{~mol})$ in minimum amount of acetic acid.

\section{3-(2-(diethylaminoethyl)-5-(2-methylbenzylidene)-2- thioxothiazolidin-4-one (D9)}

The title compound was synthesized as per the procedure for compound D1 by taking 3-(2-(diethylamino)ethyl)-2thioxothiazolidin-4-one $(0.5 \mathrm{~g}, 0.0021 \mathrm{~mol}), 2$-methylbenzaldehyde $(0.252 \mathrm{~g}, 0.0021 \mathrm{~mol})$ and ammonium acetate $(0.32 \mathrm{~g}, 0.0042 \mathrm{~mol})$ in the minimum amount of acetic acid.

\section{3-(2-(diethylamino)ethyl)-5-(4-isopropylbenzylidene)-2- thioxothiazolidin-4-one (D10)}

The title compound was synthesized as per the procedure for compound D1 by taking 3-(2-(diethylamino) ethyl)-2thioxothiazolidin-4-one $(0.5 \mathrm{~g}, \quad 0.0021 \mathrm{~mol}), \quad 4$-isopropylbenzaldehyde $(0.32 \mathrm{ml}, 0.0021 \mathrm{~mol})$ and ammonium acetate $(0.32 \mathrm{~g}$, $0.0042 \mathrm{~mol}$ ) in the minimum amount of acetic acid.

\section{Biological assays}

\section{Antifungal assay}

\section{Test fungal pathogens}

Fungal cultures used in the study were Aspergillus niger, Candida albicans, Candida parapsilosis,, Candida tropicalis and Candida glabrata. All the strains used were MTCC and were procured from Microbial Type Culture Collection and Gene Bank, CSIR-IMTECH, Chandigarh, India.

\section{Sub-culturing of test organisms}

All reference fungal cultures were subcultured on potato dextrose agar. The fungal slant was incubated for $48 \mathrm{~h}$ at $30{ }^{\circ} \mathrm{C}$. Mcfarland density ( 0.5 on the Mcfarland scale) of fungal culture was adjusted in normal saline to achieve the final concentration of $1 \times 10^{5} \mathrm{cfu} / \mathrm{ml}$ of each test organism individually. In the case of Aspergillus niger, conidial suspensions were harvested after isolates were subcultured on PDA at $30{ }^{\circ} \mathrm{C}$ to $7 \mathrm{~d}$ and were suspended in normal saline. Aspergillus niger inocula were then prepared spectrophotometrically and further diluted in normal saline to obtain a final inoculum concentration of $1 \times 10^{5} \mathrm{cfu} / \mathrm{ml}$. This had been used as adjusted inoculum for all the further studies.

\section{Determination of MIC}

MIC was done by broth microdilution method conferring to the reference of Clinical and Laboratory Standards Institute 2012, USA, using 96 well ELIZA plates [45]. To determine MIC, the compounds and the standard drug Ketoconazole (reference antimycotic drug) were dissolved in DMSO to give a stock concentration of $1 \mathrm{mg} / \mathrm{ml}$. From these serial dilutions of the test, compounds were prepared in appropriate concentrations ranging from 0.5 to $1000 \mu \mathrm{g} / \mathrm{ml}$. Each well was inoculated with $50 \mu \mathrm{l}$ of fungal suspension to give a final concentration of $1 \times 10^{5} \mathrm{cfu} / \mathrm{ml}$. The microtitre plates were incubated at $37{ }^{\circ} \mathrm{C}$ for $48 \mathrm{~h}$. The fungal growth was measured by taking absorbance at $600 \mathrm{~nm}$ using a microtitre plate reader. $0.5 \%$ DMSO and sterile RPMI medium were used as blank control, which does not inhibit the growth of fungus. MIC was defined as the lowest concentration of drug showing no growth. The experiment was performed in triplicate.

\section{Minimum fungicidal concentration (MFC)}

MFC was determined as the lowest concentration of compound that that kills $99.9 \%$ of the fungal cells after $24 \mathrm{~h}$ incubation at $37^{\circ} \mathrm{C}$. MFC values were determined by removing $100 \mu \mathrm{l}$ of fungal suspension from culture demonstrating no visible growth in MIC experiment and inoculating in fresh nutrient agar plates. Plates were incubated at $37{ }^{\circ} \mathrm{C}$ for a total period of $24 \mathrm{~h}$. The MFC is determined with the wells whose concentrations are greater than MIC. Each experiment was repeated at least 3 times

\section{Antibacterial assay}

\section{Test bacterial pathogens}

The bacterial pathogens used in the study were gram-positive bacteria Bacillus cereus, Staphylococcus aureus, gram-negative bacteria, Escherichia coli, Pseudomonas aeruginosa and Klebsiella pneumoniae. All the strains used were MTCC and procured from Microbial Type Culture Collection and Gene Bank, CSIR-IMTECH, Chandigarh, India.

\section{Sub-culturing of test organism}

All bacterial reference cultures were subcultured on nutrient agar. The bacterial slants were incubated overnight at $37{ }^{\circ} \mathrm{C}$. Mcfarland density ( 0.5 on the McFarland scale) of bacterial culture was adjusted in normal saline to achieve the final concentration of $1 \mathrm{x}$ $10^{5} \mathrm{cfu} / \mathrm{ml}$ of each test organism individually.

\section{Determination of MIC}

MIC was done by broth microdilution method recommended by the National Committee for Clinical Laboratory Standards Institute 2012 USA using 96 well ELIZA plates [46]. To determine the MIC, the compounds and standard drug ampicillin were dissolved in 
$0.5 \%$ DMSO to give a stock concentration of $1000 \mu \mathrm{g} / \mathrm{ml}$. Colony suspensions equivalent to $0.5 \mathrm{McFarland}$ standard were prepared and inoculated to yield an inoculum of $1 \times 10^{5} \mathrm{cfu} / \mathrm{ml}$. The microtitre plates were incubated at $37^{\circ} \mathrm{C}$ for $24 \mathrm{~h}$. The MIC was defined as the lowest concentration of drug showing no growth. MIC was attained from there independent tests that were performed in triplicate.

\section{Minimal bactericidal concentration (MBC)}

MBC was determined as the lowest concentration of compounds that kills $99.9 \%$ of the bacterial cells after $24 \mathrm{~h}$ incubation at $37{ }^{\circ} \mathrm{C}$. MBC values were determined by removing $100 \mu \mathrm{l}$ of bacterial suspension from culture demonstrating no visible growth in MIC experiment and inoculating in fresh nutrient agar plates. Plates were incubated at $37^{\circ} \mathrm{C}$ for a total period of $24 \mathrm{hr}$. The MBC is determined with the wells whose concentrations are greater than MIC. Each experiment was repeated at least 3 times.

\section{RESULTS AND DISCUSSION}

\section{Chemistry}

The designed molecular framework i.e. 5-benzylidene 3-(dialkylamino) alkyl-2-thioxothiazolidin-4-one has been synthesised as per scheme 1 .

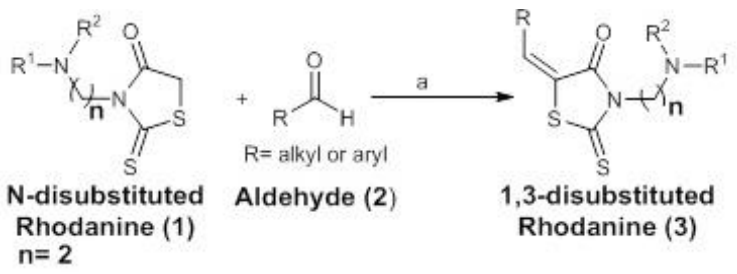

Scheme 1: Reagents and condition: a) Ammonium acetate, Acetic acid, $80-85^{\circ} \mathrm{C}$

Table 2: Synthesised derivatives

\begin{tabular}{llll}
\hline Compound code & NR $^{\mathbf{1}} \mathbf{R}^{\mathbf{2}}$ & $\mathbf{n}$ & R \\
\hline D1 & dimethyl amino & 2 & Phenyl \\
D2 & dimethyl amino & 4-ethyl,-phenyl \\
D3 & dimethyl amino & 4-methyl phenyl \\
D4 & diethyl amino & 2 & $4-$ ethyl phenyl \\
D5 & dimethyl amino & 2 & $2-$ methyl phenyl \\
D6 & dimethyl amino & 2 & 4 -isopropyl phenyl \\
D7 & diethyl amino & 2 & Phenyl \\
D8 & diethyl amino & 2 & $4-$ methyl phenyl \\
D9 & diethyl amino & 2 & $2-$ methyl phenyl \\
D10 & diethyl amino & 2 & 4 -isopropyl phenyl \\
\hline
\end{tabular}

\section{Spectral characterization of synthesised compounds}

5-Benzylidene-3-(2-(dimethylamino) ethyl)-2-thioxo-thiazolidin4-one (D1)

Brown solid (Yield 66\%), mp $105^{\circ} \mathrm{C}$; IR (KBr) $\vee\left(\mathrm{cm}^{-1}\right): 2932,2851$, $1701,1236,1175,1120 ;{ }^{1} \mathrm{H}$ NMR $(500 \mathrm{MHz}, \mathrm{DMSO}): \delta 2.36(6 \mathrm{H}, \mathrm{s})$, 2.69-2.72 (2H, t), 4.23-4.26 (2H, t), 7.35-7.40 $(4 \mathrm{H}, \mathrm{m}), 7.63(1 \mathrm{H}, \mathrm{s})$, 7.80-7.84 (1H, m); ${ }^{13} \mathrm{C}(125 \mathrm{MHz}, \mathrm{DMSO}): \delta 193.55(\mathrm{C}=\mathrm{S}), 167.87$ $(\mathrm{C}=0), 139.30,133.36,133.22,130.74,130.64,129.61,129.34$, 123.04, 114.07, 55.33, 45.34, 41.97, 29.70; HRMS (m/z) calculated for $\mathrm{C}_{14} \mathrm{H}_{16} \mathrm{~N}_{2} \mathrm{OS}_{2}$ : 292.0704, found: $293.0777\left(\mathrm{MH}^{+}\right)$.

3-(2-(dimethylamino) ethyl)-5-(4-ethylbenzylidene)-2-thioxothiazolidin-4-one (D2)

Dark brown solid (Yield 65\%), mp $96{ }^{\circ} \mathrm{C}$; IR (KBr) $v\left(\mathrm{~cm}^{-1}\right): 2928$, 2859, 1563, 1328, 1282, 1235, 1231; ${ }^{1} \mathrm{H}$ NMR (500 MHz, DMSO): $\delta 1.19(3 \mathrm{H}, \mathrm{bs}), 2.30(6 \mathrm{H}, \mathrm{s}), 2.61-2.67(4 \mathrm{H}, \mathrm{m}), 4.20-4.23(2 \mathrm{H}, \mathrm{t})$, 7.19-7.23 (2H, m), 7.27-7.33 (2H, m), $7.62(1 \mathrm{H}, \mathrm{s}) ;{ }^{13} \mathrm{C}(125 \mathrm{MHz}$, DMSO): $\delta 192.02(\mathrm{C}=\mathrm{S}), 167.92(\mathrm{C}=0), 133.43,130.87,129.98$, $128.93,128.55,121.80,55.16,45.12,41.76,28.92,15.12$; HRMS (m/z) calculated for $\mathrm{C}_{16} \mathrm{H}_{20} \mathrm{~N}_{2} \mathrm{OS}$ : 320.1017 , found: $321.1086\left(\mathrm{MH}^{+}\right)$.

3-(2-(dimethylamino)ethyl)-5-(4-methylbenzylidene)-2thioxothiazolidin-4-one (D3)

Yellow coloured solid (Yield 70\%), mp $102{ }^{\circ} \mathrm{C}$; IR (KBr) $v\left(\mathrm{~cm}^{-1}\right)$ : 2917, 2855, 2786, 1701, 1590, 1334, 1180; ${ }^{1} \mathrm{H}$ NMR $(500 \mathrm{MHz}$, DMSO), $\delta 2.25(6 \mathrm{H}, \mathrm{s}), 2.33(3 \mathrm{H}, \mathrm{s}), 2.56-2.59(2 \mathrm{H}, \mathrm{t}), 4.17-4.20(2 \mathrm{H}$, t), 7.20-7.21 (2H, d), 7.31-7.32 (2H, d), $7.63(1 \mathrm{H}, \mathrm{s}) ;{ }^{13} \mathrm{C}(125 \mathrm{MHz}$, DMSO): $\delta 193.57(\mathrm{C}=\mathrm{S}), 167.91 \quad(\mathrm{C}=0), 141.63,133.35,130.74$, 130.67, 130.13, 121.81, 55.53, 45.61, 42.27, 21.64; HRMS (m/z) calculated for $\mathrm{C}_{15} \mathrm{H}_{18} \mathrm{~N}_{2} \mathrm{OS}$ : 306.0861 found: 307. $0.0930\left(\mathrm{MH}^{+}\right)$.

\section{3-(2-(diethylamino)ethyl)-5-(4-ethyl benzylidene)-2-thioxo-} thiazolidin-4-one (D4)

Yellow coloured solid (Yield 68\%), mp $108{ }^{\circ} \mathrm{C}$; IR (KBr) $\vee\left(\mathrm{cm}^{-1}\right)$ : 2928, 2855, 1699, 1594, 1283; ${ }^{1} \mathrm{H}$ NMR (500 mHz, DMSO), $\delta 0.98-$ $1.01(6 \mathrm{H}, \mathrm{t}), 1.18-1.19(3 \mathrm{H}, \mathrm{t}), 2.55-2.74(10 \mathrm{H}=2 \mathrm{H} \times 5, \mathrm{~m}), 7.23-7.24$ $(2 \mathrm{H}, \mathrm{d}), 7.34-7.35(2 \mathrm{H}, \mathrm{d}), 7.63(1 \mathrm{H}, \mathrm{s}) ;{ }^{13} \mathrm{C}(125 \mathrm{MHz}, \mathrm{DMSO}) ; \delta$ $192.04(\mathrm{C}=\mathrm{S}), 167.87$ ( $\mathrm{C}=0), 147.84,133.27,130.89,128.94,121.87$,
48.36, 47.37, 42.01, 28.92, 15.26, 15.14, 14.12, 11.91; HRMS (m/z) calculated for $\mathrm{C}_{18} \mathrm{H}_{24} \mathrm{~N}_{2} \mathrm{OS}_{2}$ : 348.1330 , found: $349.1415\left(\mathrm{MH}^{+}\right)$.

3-(2-(dimethylamino)ethyl)-5-(2-methylbenzylidene-2thioxothiazolidin-4-one (D5)

Dark brown coloured solid (Yield 68\%); mp $115^{\circ} \mathrm{C}$; IR (KBr) $\vee(\mathrm{cm}$ 1): 2627, 1725, 1431, 1356, 1310, 1291; ${ }^{1} \mathrm{H}$ NMR (500 MHz, DMSO): $\delta 2.27(6 \mathrm{H}, \mathrm{s}), 2.39(3 \mathrm{H}, \mathrm{s}), 2.58-2.61(4 \mathrm{H}, \mathrm{m}), 7.19-7.33(4 \mathrm{H}, \mathrm{m}), 7.88$ $(1 \mathrm{H}, \mathrm{s}),{ }^{13} \mathrm{C}(125 \mathrm{MHz}, \mathrm{DMSO}): \delta 194.10(\mathrm{C}=\mathrm{S}), 167.61$ (C=0), 139.47, 132.43, 131.17, 131.04, 130.71, 128.07, 126.70, 124.23, 55.50, 45.55, 42.18, 29.70, 19.99; HRMS (m/z) calculated for $\mathrm{C}_{15} \mathrm{H}_{18} \mathrm{~N}_{2} \mathrm{OS}_{2}$ : 306.0861, found: $307.0938\left(\mathrm{MH}^{+}\right)$.

\section{3-(2-(dimethylamino)ethyl)-5-(4-isopropylbenzylidene)-2- thioxothiazolidin-4-one (D6)}

Light brown coloured solid (Yield 70\%); mp $110^{\circ} \mathrm{C}$; IR (KBr) v (cm 1): 2966, 2924, 2790, 1703, 1592, 1326; ${ }^{1} \mathrm{H}$ NMR (500 MHz, DMSO); $\delta$ 1.21-1.22 (6H, d), $2.26(6 \mathrm{H}, \mathrm{s}), 2.58-2.61(2 \mathrm{H}, \mathrm{t}) 2.87-2.92(2 \mathrm{H}, \mathrm{m})$, 4.84-4.94 $(1 \mathrm{H}, \mathrm{m}), 7.26-7.27(2 \mathrm{H}, \mathrm{d}), 7.35-7.36(2 \mathrm{H}, \mathrm{d}), 7.64(1 \mathrm{H}, \mathrm{s})$; ${ }^{13} \mathrm{C}$ (125 MHz, DMSO): $\delta 192.04$ (C=S), 167.95 (C=0), 152.41, 133.39, $131.01,130.93,130.02,127.54,127.15,114.08,55.47,45.53,42.19$, 34.48, 34.22, 23.64; HRMS (m/z) calculated for $\mathrm{C}_{17} \mathrm{H}_{22} \mathrm{~N}_{2} \mathrm{OS}_{2}$ : 334.1174, found: $335.1249\left(\mathrm{MH}^{+}\right)$

\section{5-benzyliden-3-(2-diethylamino)ethyl)-2-thioxothiazolidin-4-} one (D7)

Dark brown coloured solid (Yield 68\%); mp $125^{\circ} \mathrm{C}$; IR (KBr) $v(\mathrm{~cm}$ 1): 2928, 2855, 1699, 1592, 1283, 1176; ${ }^{1} \mathrm{H}$ NMR ( $500 \mathrm{MHz}$, DMSO), 81.01-1.04 (6H, t), 2.62-2.66 (4H, m), 2.77-2.80 (4H, m) 7.19-7.24 $(1 \mathrm{H}, \mathrm{m}), 7.40-7.41(4 \mathrm{H}, \mathrm{m}), 7.65(1 \mathrm{H}, \mathrm{s}) ;{ }^{13} \mathrm{C}(125 \mathrm{mHz}, \mathrm{DMSO}) ; \delta$ $193.45(\mathrm{C}=\mathrm{S}), 167.76(\mathrm{C}=0), 139.29,133.13,129.34,127.91,123.06$, 48.02, 47.16, 41.62, 11.52; HRMS (m/z) calculated for $\mathrm{C}_{14} \mathrm{H}_{20} \mathrm{~N}_{2} \mathrm{OS}$ : 320.1017, found: $321.1083\left(\mathrm{MH}^{+}\right)$.

\section{3-(2-(diethylamino)ethyl)-5-(4-methylbenzylidene)-2-thioxo-} thiazolidin-4-one (D8)

Yellow coloured solid (Yield 68\%); mp $112{ }^{\circ} \mathrm{C}$; IR $(\mathrm{KBr}) \vee\left(\mathrm{cm}^{-1}\right)$ : 2928, 2855, 1699, 1592, 1282; ${ }^{1} \mathrm{H}$ NMR (500MHz, DMSO); $\delta 0.96-0.99$ $(6 \mathrm{H}, \mathrm{t}), 2.33-2.71(11 \mathrm{H}=3 \mathrm{H}+2 \mathrm{H}+2 \mathrm{H}+4 \mathrm{H}, \mathrm{m}), 7.19-7.22(2 \mathrm{H}, \mathrm{t}), 7.31-$ 
$7.33(2 \mathrm{H}, \mathrm{d}) ; 7.62(1 \mathrm{H}, \mathrm{s}) ;{ }^{13} \mathrm{C}(125 \mathrm{MHz}, \mathrm{DMSO}): \delta 193.54(\mathrm{C}=\mathrm{S})$, $167.88(\mathrm{C}=0), 141.61,133.20,130.76,130.13,114.08,48.55,47.47$, 42.24, 21.64, 12.11; HRMS (m/z) calculated for $\mathrm{C}_{17} \mathrm{H}_{22} \mathrm{~N}_{2} \mathrm{OS}_{2}$ : 334.1174, found: $335.1259\left(\mathrm{MH}^{+}\right)$.

\section{3-(2-(diethylaminoethyl)-5-(2-methylbenzylidene)-2- thioxothiazolidin-4-one (D9)}

Yellow coloured solid (Yield 70\%); mp $112{ }^{\circ} \mathrm{C}$; IR $(\mathrm{KBr}) \vee\left(\mathrm{cm}^{-1}\right)$ : $2928,2851,1699,1594,1283 ;{ }^{1} \mathrm{H}$ NMR $(500 \mathrm{MHz}, \mathrm{DMSO}) ; \delta 0.97-$ $1.00(6 \mathrm{H}, \mathrm{t}), 2.39(3 \mathrm{H}, \mathrm{s}), 2.52-2.71(8 \mathrm{H}=4 \mathrm{H}+4 \mathrm{H}, \mathrm{m}), 7.19-7.25(3 \mathrm{H}$, $\mathrm{m}), 7.25-7.34(1 \mathrm{H}, \mathrm{m}), 7.84(1 \mathrm{H}, \mathrm{s}) ;{ }^{13} \mathrm{C}(125 \mathrm{MHz}, \mathrm{DMSO}): \delta 194.05$ $(\mathrm{C}=\mathrm{S}), 167.57$ ( $\mathrm{C}=0), 139.46,132.44,128.07,126.71,114.08,48.56$, $47.53,42.26,20.00,12.15$; HRMS (m/z) calculated for $\mathrm{C}_{17} \mathrm{H}_{22} \mathrm{~N}_{2} \mathrm{OS}_{2}$ : 334.1174, found: $335.1249\left(\mathrm{MH}^{+}\right)$.

\section{3-(2-(diethylamino)ethyl)-5-(4-isopropylbenzylidene)-2-} thioxothiazolidin-4-one (D10)

Light brown coloured solid (Yield 65\%); mp $118^{\circ} \mathrm{C}$; IR (KBr) $v\left(\mathrm{~cm}^{-1}\right)$ : $2932,2920,2851,1699,1593,1283,1175 ;{ }^{1}$ H NMR (500 MHz, DMSO), $\delta 0.98(6 \mathrm{H}, \mathrm{s}), 1.18-1.21(6 \mathrm{H}, \mathrm{t}), 2.54-2.88(8 \mathrm{H}=4 \mathrm{H}+2 \mathrm{H}+2 \mathrm{H}, \mathrm{m}), 4.83-$ $4.94(1 \mathrm{H}, \mathrm{m}), 7.26-7.37(4 \mathrm{H}, \mathrm{m}), 7.63(1 \mathrm{H}, \mathrm{s}) ;{ }^{13} \mathrm{C}(125 \mathrm{MHz}, \mathrm{DMSO}): \delta$ 193.57 (C=S), 167.89 (C=0), 152.39, 141.14, 133.20, 130.93, 127.54, $121.91,48.51,47.45,42.21,34.22,23.64,12.08$; HRMS (m/z) calculated for $\mathrm{C}_{19} \mathrm{H}_{26} \mathrm{~N}_{2} \mathrm{OS}_{2}: 362.1487$, found: $363.1571\left(\mathrm{MH}^{+}\right)$.

\section{Antifungal activity}

All the compounds were screened for their in vitro antifungal activity against various species of Candida and Aspergillus niger. Ketoconazole was taken as the reference standard. Compounds with MIC $>250 \mu \mathrm{g} / \mathrm{ml}$ were considered as inactive. MIC between 250-125 $\mu \mathrm{g} / \mathrm{ml}$ was indicative of low activity and MIC between 64 to $32 \mu \mathrm{g} / \mathrm{ml}$ exhibited moderate activity. MIC less than $16 \mu \mathrm{g} / \mathrm{ml}$ can be considered as the activities which are used in the clinical situation. The MIC values of the compounds (D1-D10) against fungal pathogens are given in table 3. D10 was observed to show MIC value at $16 \mu \mathrm{g} / \mathrm{ml}$ against Candida glabrata. Similarly, D6 also showed MIC value at $16 \mu \mathrm{g} / \mathrm{ml}$ against Candida tropicalis and are considered as lead compounds for further development. The MFC values of the compounds against fungal pathogens are given in table 4. Compound D10 was observed to show MFC value at $32 \mu \mathrm{g} / \mathrm{ml}$ against Candida glabrata and compound D6 showed MFC value at $32 \mu \mathrm{g} / \mathrm{ml}$ against Candida tropicalis The structure-activity relationship studies revealed that the most potent compounds D6 (3-(2(dimethylamino)ethyl)-5-(4-isopropylbenzylidene)-2-

thioxothiazolidin-4-one)and D10(3-(2-(diethylamino) ethyl)-5-(4isopropyl benzylidene)-2-thioxothiazolidin-4-one) which are having 4 -isopropyl benzylidene group at $5^{\text {th }}$ position of rhodanine showed MIC value $16 \mu \mathrm{g} / \mathrm{ml}$ against candida albicans and Candida glabrata respectively.

Table 3: Minimum Inhibitory concentration of derivatives of D1-D10 against fungal pathogens

\begin{tabular}{|c|c|c|c|c|c|c|c|c|c|c|c|}
\hline \multicolumn{12}{|c|}{ Minimum Inhibitory concentration $(\mu \mathrm{g} / \mathrm{ml})^{*}$} \\
\hline Fungal pathogens & D1 & D2 & D3 & D4 & D5 & D6 & D7 & D8 & D9 & D10 & ketoconazole \\
\hline Aspergillusniger & 32 & 125 & 125 & 32 & 32 & 64 & 32 & 125 & 64 & 125 & 1 \\
\hline Candida albicans & 32 & 32 & 125 & 32 & 32 & 32 & 32 & 64 & 32 & 32 & 0.5 \\
\hline Candida glabrata & 64 & 64 & 125 & 64 & 32 & 64 & 32 & 64 & 64 & 16 & 0.5 \\
\hline Candida parapsilosis & 32 & 64 & 125 & 64 & 32 & 34 & 32 & 125 & 64 & 64 & 0.5 \\
\hline Candida tropicalis & 64 & 64 & 125 & 32 & 32 & 16 & 32 & 32 & 32 & 64 & 1 \\
\hline
\end{tabular}

*Values are average of three readings

Table 4: Minimum fungicidal concentration of derivatives of D1-D10 against fungal pathogens

\begin{tabular}{|c|c|c|c|c|c|c|c|c|c|c|}
\hline \multicolumn{11}{|c|}{ Minimum fungicidal concentration $(\mu \mathrm{g} / \mathrm{ml})^{*}$} \\
\hline Fungal pathogens & D1 & D2 & D3 & D4 & D5 & D6 & D7 & D8 & D9 & D10 \\
\hline Aspergillus niger & 64 & 250 & 250 & 64 & 64 & 125 & 64 & 250 & 125 & 250 \\
\hline Candida albicans & 64 & 64 & 250 & 64 & 64 & 64 & 64 & 125 & 64 & 64 \\
\hline Candida glabrata & 125 & 125 & 250 & 125 & 64 & 125 & 64 & 125 & 125 & 32 \\
\hline Candida parapsilosis & 64 & 125 & 250 & 125 & 64 & 125 & 64 & 250 & 125 & 125 \\
\hline Candida tropicalis & 125 & 125 & 250 & 64 & 64 & 32 & 64 & 64 & 64 & 125 \\
\hline
\end{tabular}

*Values are average of three readings

\section{Antibacterial activity}

The MIC values of the compounds (D1-D10) and the positive control Ampicillin against the gram-positive bacterial strains (Bacillus cereus and Staphylococcus aureus) and gram-negative strains (Escherichia coli, Klebsiella pneumoniae, and Pseudomonas. aeruginosa) are given. The synthesized compounds (D1-D10, table 5) were also been evaluated for their antibacterial activities against different species. Ampicillin was taken as the reference standard. Compounds with MIC $>250 \mu \mathrm{g} / \mathrm{ml}$ were considered as inactive and MIC between $250 \mathrm{and} 125 \mu \mathrm{g} / \mathrm{ml}$ were indicative of low activity. MIC between 64 to $32 \mu \mathrm{g} / \mathrm{ml}$ showed moderate activity. MIC less than $16 \mu \mathrm{g} / \mathrm{ml}$ was supposed to be the activity which can be used in the clinical situation. In table 4, D2 elicited a moderate antibacterial activity at MIC value of $32 \mu \mathrm{g} / \mathrm{ml}$ in gram-negative strains, i. e, against Escherichia coli and Klebsiella pneumoniae. Compound D5 showed activity against Klebsiella pneumoniae at MIC value of $32 \mu \mathrm{g} / \mathrm{ml}$. But other compounds were observed to be rather resistant towards gram-positive strains. In comparison with Ampicillin which gave a MIC value of $1 \mu \mathrm{g} / \mathrm{ml}$ in Escherichia coli, Klebsiella pneumonia, the results obtained with D2 and D5 were not very significant. The MBC values of the compounds (D1-D10) against both gram-positive and gram-negative strains are given in table 6 . The MBC values of all the compounds were $\geq 64 \mu \mathrm{g} / \mathrm{ml}$ against all bacterial strains.

Table 5: Minimum inhibitory concentration $(\mu \mathrm{g} / \mathrm{ml})$ of derivatives of D1-D10 against bacterial strains

\begin{tabular}{|c|c|c|c|c|c|c|c|c|c|c|c|}
\hline \multicolumn{12}{|c|}{ Minimum inhibitory concentration $(\mu \mathrm{g} / \mathrm{ml})^{*}$} \\
\hline Pathogens & D1 & D2 & D3 & D4 & D5 & D6 & D7 & D8 & D9 & D10 & Ampicillin \\
\hline Bacillus cereus & 64 & 125 & 250 & 125 & 64 & 125 & 64 & 125 & 125 & 125 & 2 \\
\hline Staphylococcus aureus & 64 & 62.5 & 250 & 125 & 64 & 125 & 64 & 125 & 125 & 125 & 0.5 \\
\hline Escherichia coli & 64 & 32 & 125 & 125 & 64 & 64 & 64 & 125 & 125 & 125 & 1 \\
\hline Klebsiella pneumoniae & 64 & 32 & 250 & 125 & 32 & 64 & 64 & 125 & 125 & 125 & 1 \\
\hline Pseudomonas aeruginosa & 64 & 250 & 250 & 250 & 250 & 250 & 250 & 250 & 250 & 250 & 1 \\
\hline
\end{tabular}

*Values are average of three readings 
Table 6: Minimum bactericidal concentration $(\mu \mathrm{g} / \mathrm{ml})$ of derivatives of D1-D10 against bacterial strains

\begin{tabular}{|c|c|c|c|c|c|c|c|c|c|c|}
\hline \multicolumn{11}{|c|}{ Minimum bactericidal concentration $(\mu \mathrm{g} / \mathrm{ml})^{*}$} \\
\hline Pathogens & D1 & D2 & D3 & D4 & D5 & D6 & D7 & D8 & D9 & D10 \\
\hline Bacillus cereus & 125 & 250 & 500 & 250 & 125 & 250 & 125 & 250 & 250 & 250 \\
\hline Staphylococcus aureus & 125 & 125 & 500 & 250 & 125 & 250 & 125 & 250 & 250 & 250 \\
\hline Escherichia coli & 125 & 64 & 250 & 250 & 125 & 125 & 125 & 250 & 250 & 250 \\
\hline Klebsiella pneumoniae & 125 & 64 & 500 & 250 & 64 & 125 & 125 & 250 & 250 & 250 \\
\hline Pseudomonas aeruginosa & 125 & 500 & 500 & 500 & 500 & 500 & 500 & 500 & 500 & 500 \\
\hline
\end{tabular}

*Values are average of three readings

Structural modifications of rhodanine derivatives results in compounds with a broad spectrum of pharmacological activities [47]. Substitution of various benzylidene derivatives at $5^{\text {th }}$ position increases hydrophobicity and led to an increase in antimicrobial activity [48]. It has been reported that, the presence of the hydrophobic phenylalanine side chain at the N3-position and the electron-deficient benzylidene moiety at the C5-position of the rhodanine scaffold is responsible for the antibacterial activity of these compounds against methicillin-resistant Staphylococcus aureus [49]. In our study 4-ethylbenzylidene and 2methylbenzylidene group at $5^{\text {th }}$ position of rhodanine showed good antibacterial activity at $32 \mu \mathrm{g} / \mathrm{ml}$. A series of benzylidenerhodanines were reported to be acting as antifungal agents which are most active against Candida genus and Candida neoformans [50]. In our study, 4-isopropyl benzylidene group at $5^{\text {thposition of }}$ rhodanine showed MIC value $16 \mu \mathrm{g} / \mathrm{ml}$ against candida albicans and Candida glabrata, respectively.

\section{CONCLUSION}

In the search for a novel antimicrobial agent different 5-benzylidene substituted rhodanine derivatives were synthesized from various $\mathrm{N}$ substituted rhodanine derivatives. All the ten synthesized compounds were screened for antimicrobial activity using the microdilution method recommended by the National Committee for Clinical Laboratory Standards, USA (CLSI 2006) to assess their antimicrobial activity. All the compounds exhibited good antifungal activity and compound D6(3-(2-(dimethylamino)ethyl)-5-(4-isopropyl benzylidene)-2-thioxothiazolidin-4-one) and D10(3-(2-(diethylamino) ethyl)-5-(4-isopropyl benzylidene)-2 thioxothiazolidin-4-one) which are having 4-isopropyl benzylidene group at $5^{\text {th }}$ position of rhodanine showed MIC value $16 \mu \mathrm{g} / \mathrm{ml}$ against candida tropicalis and Candida glabrata respectively. Compounds D2 and D5 Showed good antibacterial activity at $32 \mu \mathrm{g} / \mathrm{ml}$. All the other compounds showed moderate antibacterial activity. Based on these results, it can be suggested these compounds may lead to the development of more potent antimicrobial agents in the future.

\section{ACKNOWLEDGEMENT}

I sincerely thank Dr. Lakshmi Nair for the scientific discussion on antimicrobial studies and Ms. Reshmi Ramakrishnan for the editing support for this paper.

\section{FUNDING}

This work was financially supported by State Board of Medical Research, Medical College, Thiruvananthapuram (Grant No: 5121/2015).

\section{AUTHORS CONTRIBUTIONS}

All the authors have contributed equally.

\section{CONFLICT OF INTERESTS}

Authors declare no conflict of interest.

\section{REFERENCES}

1. Khameneh B, Diab R, Ghazvini K. Breakthroughs in bacterial resistance mechanisms and the potential ways to combat them. Microb Pathog 2016;95:32-42.

2. Silveira AD, Perez A. Liposomes as novel anti-infectives targeting bacterial virulence factors? Expert Rev Anti Infect Ther 2015;13:531-3.
3. Song B, Wen S. Development of quorum-based anti-virulence therapeutics targeting gram-negative pathogens. Int J Mol Sci 2013;14:16570-99.

4. Cascioferro S, Cusimano MG, Schillaci D. Anti adhesion agents against gram-positive pathogens. Future Microbiol 2014;9:1209-20.

5. Rademacher J, Welte T. New antibiotics-standstill or progress. Med Klin Intensivmed Notfmed 2017;112:206-13.

6. Boucher HW, Talbot GH, Benjamin DK, Bradley J, Guidos RJ, Jones RN, et al. $10 \times 20$ progress-development of new drugs active against gram-negative bacilli: an update from the infectious diseases society of America. Clin Infectious Dis 2013;56:1685-94.

7. Mendgen T, Steuer C, Klein CD. Privileged scaffolds or promiscuous binders: a comparative study on rhodanines and related heterocycles in medicinal chemistry. J Med Chem 2012;55:743-53.

8. Brown FC. 4-Thiazolidinones. Chem Rev 1961;61:463-521.

9. Tomasic T, Masic LP. Rhodanine as a scaffold in drug discovery: a critical review of its biological activities and mechanisms of target modulation. Expert Opin Drug Discovery 2012;7:549-60.

10. Tomasic T, Masic LP. Rhodanine as a privileged scaffold in drug discovery. Cmc 2009;16:1596-629.

11. Tomasic T, Masic LP. Chemistry and reactivity of rhodanines. In: S Brase. Privileged scaffolds in medicinal chemistry: design, synthesis, evaluation. Cambridge: Royal Society of Chemistry; 2015. p. 214-31.

12. Singh SP, Parmar SS, Raman K, Stenberg VI. Chemistry and biological activity of thiazolidinones. Chem Rev 1981;81:175203.

13. Hajibabaei K. 2-Thioxothiazolidin-4-one (Rhodanine). Synlett 2014;25:2083-4.

14. Pardasani R, Pardasani P, Sherry D, Chaturvedi V. Synthetic and antibacterial studies of rhodanine derivatives with indol-2, 3diones. Ind J Chem-B 2001;40:1275-8.

15. Song MX, Zheng CJ, Deng XQ, Wang Q, Hou SP, Liu TT, et al. Deng, synthesis and bioactivity evaluation of rhodanine derivatives as potential anti-bacterial agents. Euro J Med Chem 2012;54:403-12.

16. Tejchman W, Korona Glowniak I, Malm A, Zylewski M, Suder P. Antibacterial properties of 5-substituted derivatives of rhodanine3-carboxyalkyl acids. Med Chem Res 2017;26:1316-24.

17. Opperman TJ, Kwasny SM, Williams JD. Aryl rhodanines specifically inhibit staphylococcal and enterococcal biofilm formation. Antimicrob Agents Chemother 1995;53:4357-67.

18. Grant EB, Guiadeen D, Baum EZ, Foleno BD, Jin H, Montenegro DA, et al. The synthesis and SAR of rhodanines as novel class $C \beta$ lactamase inhibitors. Bioorg Med Chem Lett 2000;10:2179-82.

19. Miao J, Zheng CJ, Sun LP, Song MX, Xu LL, Piao HR. Synthesis and potential antibacterial activity of new rhodanine-3-acetic acid derivatives. Med Chem Res 2013;22:4125-32.

20. Inamori $Y$, Okamoto $Y$, Takegawa $Y$, Tsujibo H, Sakagami $Y$, Kumeda $\mathrm{Y}$, et al. Insecticidal and antifungal activities of aminorhodanine derivatives. Biosci Biotechnol Biochem 1998;62:1025-7.

21. Marc G, Ionuţ I, Pirnau A, Vlase L. Microwave-assisted synthesis of 3,5-disubstituted thiazolidine-2,4-diones with antifungal activity. Design, synthesis, virtual and in vitro Antifungal Screening. Int J Farmacia 2017;65:414-22.

22. $\mathrm{Xu} \mathrm{H}$, Wang YY. Antifungal agents. Part 5:Synthesis and antifungal activities of aminoguanidine derivatives of $\mathrm{N}$-arylsulfonyl-3acylindoles. Bioorg Med Chem Lett 2010;20:7274-7. 
23. Terashima H, Hama K, Yamamoto R, Tsuboshima M, Kikkawa R, Hatanaka I, et al. Effects of a new aldose reductase inhibitor on various tissues in vitro. J Pharmacol Exp Ther 1984;229:22630.

24. Bajaj M, Suraamornkul S, Hardies LJ, Glass L, Musi N, DeFronzo RA. Effects of peroxisome proliferator-activated receptor (PPAR) $-\alpha$ and PPAR- $\gamma$ agonists on glucose and lipid metabolism in patients with type 2 diabetes mellitus. Diabetologia 2007;50:1723-31.

25. Taniyama $H$, Yasui $B$, Takehara $N$, Uchida $H$. Chemotherapeutics for mycobacterium tuberculosis. XIX. Synthesis and antibacterial activity of some 3-substituted rhodanines. Yakugaku Zasshi 1959;79:1465-8.

26. Singh J, Nathan CF, Bryk R, Samy R, Pupek K, Gurney M. Cyclic carboxylic acid rhodanine derivatives for the treatment and prevention of tuberculosis. WO Pat 2008005651; 2008. Available from: https://patents.google.com/ patent/ W02008005651A2/en

27. Sim MM, Ng SB, Buss AD, Crasta SC, Goh KL, Lee SK. Benzylidene rhodanines as novel inhibitors of UDP-Nacetylmuramate/l-alanine ligase. Bioorg Med Chem Lett 2002;12:697-9.

28. Azizmohammadi M, Khoobi M, Ramazani A. 2H-chromene derivatives bearing thiazolidine-2,4-dione, rhodanine or hydantoin moieties as potential anticancer agents. Eur J Med Chem 2013;59:15-22.

29. Moorthy BT, Ravi S, Srivastava M, Chiruvella KK, Hemlal H, Joy 0 , et al. Novel rhodanine derivatives induce growth inhibition followed by apoptosis. Bioorg Med Chem Lett 2010;20:6297301.

30. Katritzky AR, Tala SR, Lu H. Design, synthesis, and structureactivity relationship of a novel series of 2-aryl 5-(4-oxo-3phenethyl-2-thioxothiazolidinylidenemethyl) furans as HIV-1 entry inhibitors. J Med Chem 2009;52:7631-9.

31. He XY, Lu L, Qiu J, Zou P, Yu F, Jiang XK, et al. Small molecule fusion inhibitors: design, synthesis and biological evaluation of (Z)-3-(5-(3-benzyl-4-oxo-2-thioxothiazolidinylidene) methyl)$\mathrm{N}$-(3-carboxy-4-hydroxy) phenyl-2, 5-dimethylpyrroles and related derivatives targeting HIV-1 gp41. Bioorg Med Chem 2013;20:7539-48.

32. Damond F, Worobey M, Campa P, Farfara I, Colin G, Matheron S, Brun Vézinet $\mathrm{F}$, et al. Identification of a highly divergent HIV type 2 and proposal for a change in HIV type 2 classification. AIDS Res Hum Retroviruses 2004;20:666-72.

33. Santiago ML, Range F, Keele BF, Li Y, Bailes E, Bibollet Ruche F, et al. Simian immunodeficiency virus infection in free-ranging sooty mangabeys (Cercocebus atys atys) from the Tai Forest, Cote d'Ivoire: implications for the origin of epidemic human immunodeficiency virus type 2. J Virol 2005;79:12515-27.

34. Keele BF, Van Heuverswyn F, Li Y. Chimpanzee reservoirs of pandemic and nonpandemic HIV-1. Science 2006;313:523-6.

35. Taylor M, Alonso Gonzalez M, Gomez B, Korenromp E, Broutet N. World health organization global health sector strategy on sexually transmitted infections: an evidence-to-action summary for Colombia. Rev Colomb Obstet Ginecol 2017;68:193-201.

36. Tintori C, Corradi V, Magnani M, Manetti F, Botta M. Targets looking for drugs: a multistep computational protocol for the development of structure-based pharmacophores and their applications for hit discovery. J Chem Inf Model 2008;48:2166-79.

37. Rinaldi M, Tintori C, Franchi L. A versatile and practical synthesis toward the development of novel HIV-1 integrase inhibitors. Chem Med Chem 2011;6:343-52.

38. Botta L, Maccari G, Calandro P. One drug for two targets: biological evaluation of antiretroviral agents endowed with antiproliferative activity. Bioorg Med Chem Lett 2017;27:2502-5.

39. Zaveri M, Kawathekar N. Synthesis and antimalarial activity of some new 3-phenyl-2-thioxothiazolidin-4-one derivatives. Int J Curr Pharm Res 2017;9:58-61.

40. Bulic B, Pickhardt M, Khlistunova I. Rhodanine-based tau aggregation inhibitors in cell models of tauopathy. Angew Chem 2007;119:9375-9.

41. Ono M, Hayashi S, Matsumura K. Rhodanine and thiohydantoin derivatives for detecting tau pathology in Alzheimer's brains. ACS Chem Neurosci 2011;2:269-75.

42. Mousavi SM, Zarei M, Hashemi SA, Babapoor A, Amani AM. A conceptual review of rhodanine: current applications of antiviral drugs, anticancer and antimicrobial activities. Artif Cells Nanomed Biotechnol 2019;47:1132-48.

43. Kaur Manjal S, Kaur R, Bhatia R. Synthetic and medicinal perspective of thiazolidinones: a review. Bioorg Chem 2017;75:406-23.

44. Xing C, Wang L, Tang X, Sham YY. Development of selective inhibitors for anti-apoptotic Bcl-2 proteins from BHI-1. Bioorg Med Chem Lett 2007; 15:2167-76.

45. National Committee for Clinical Laboratory Standards. Reference Method for Broth Dilution Antifungal Susceptibility Testing of Yeasts. $2^{\text {nd }}$ ed. Approved Standard M27-A2. Wayne, PA: NCCLS; 2002.

46. Clinical and Laboratory Standards Institute (CLSI), "Methods for dilution antimicrobial susceptibility tests for bacteria that grow aerobically, approved standard-ninth edition," CLSI Documents M07-A9, Clinical and Laboratory Standards Institute (CLSI), Wayne, PA, USA; 2012.

47. Ernst R, Roland NI, Gordon AA. Rhodanine. Org Synth 1947;27:73.

48. Tomasic T, Zidar N, Mueller Premru M, Kikelj D, Masic LP. Synthesis and antibacterial activity of 5-ylidenethiazolidin-4ones and 5-benzylidene-4,6-pyrimidinediones. Eur J Med Chem 2010;45:1667-72.

49. Hardej N, Ashby CR Jr, Khadtare NS. The synthesis of phenylalanine-derived C5-substituted rhodanines and their activity against selected methicillin-resistant staphylococcus aureus (MRSA) strains. Eur J Med Chem 2010;45:5827-32.

50. Sortino M, Delgado P, Juarez S, Quiroga J, Abonia R, Insuasty B, et al. Synthesis and antifungal activity of (Z)-5arylidenerhodanines. Bioorg Med Chem Lett 2007;15:484-94. 